\title{
Sensing Performance of Mono and Bimetallic Nano Photonics Surface Enhanced Raman Scattering (SERS) Devices
}

\author{
Doaa S. Jubair ${ }^{a *}$, Alwan M. Alwan (D) ${ }^{b}$, Walid K. Hamoudi ${ }^{c}$ \\ a Department of Applied Sciences, University of Technology, Baghdad, Iraq, 100271@uotechnology.edu.iq \\ ${ }^{\mathrm{b}}$ Department of Applied Sciences, University of Technology, Baghdad, Iraq, alkrzsm@yahoo.com \\ ${ }^{\mathrm{c}}$ Department of Medical Optics Techniques, Al-Farabi University College, Baghdad, Iraq, \\ wjuaini2007@yahoo.com \\ *Corresponding author.
}

Submitted: $19 / 01 / 2021$

Accepted: 07/03/2021

Published: 25/07/2021

\section{K E Y W O R D S}

SERS, duty cycle, bimetallic, nanocolumns, pesticide detection.

\begin{abstract}
A B S T R A C T
In this research, sensing performance of mono and bimetallic nanophotonics SERS sensors of gold-silver nano-columns for the detection of chlorpyrifos was investigated. For optimum substrates for Goldsilver/nano-column surface-enhanced, Raman scattering (SERS) was achieved with the silicon substrate. By combining the Ag SERS activity with the Au chemical stability and nano-columns Si large field enhancement, the Au-Ag/nano-columns Si substrate revealed perfect reproducibility, homogeneity, sensitivity in addition to chemical stability. The sensors were tested by Atomic force microscope (AFM), field emission scanning electron microscope (FESEM), energy-dispersive X-ray analysis $(E D S)$, and X-ray diffraction (XRD). Findings presented in this research indicated modified distributions and sizes of formed alloy nanoparticles, and the hot spots junctions within the nanophotonics layer after changing the nanoparticles types. The SERS sensors performance displayed an excellent recognition of ultra-low concentrations of chlorpyrifos solutions with an exponential relationship with the Raman signal. The highest enhancement factor $\left(E f=1.56 \times 10^{6}\right)$ and minimum limit of detection 0.069 $\mathrm{mg} / \mathrm{Kg}$ were obtained with $\mathrm{Au}$ - $\mathrm{Ag}$ sensors.
\end{abstract}

How to cite this article: D. Sulaiman, A. M. Alwan, and W. K. Hamoudi, "Sensing performance of mono and bimetallic nano photonics SERS devices," Engineering and Technology Journal, Vol. 39, No. 07, pp. 1174-1184, 2021.

DOI: https://doi.org/10.30684/etj.v39i7.1982

This is an open access article under the CC BY 4.0 license http://creativecommons.org/licenses/by/4.0

\section{INTRODUCTION}

Surface Plasmons (SPs) is a physico-chemical phenomena where collective excitation of free conductive electrons at the metal-dielectric interfaces which induced by electromagnetic field [1]. These have been provided within nanoparticle (NP) surfaces or noble metal thin films. The light with metallic nanostructures interaction study is a rapidly emerging research area called Plasmonics [2]. Plasmonic nanostructures have targeted engineering that gives the ability to manipulate and control visible wavelengths at a nanometer scale [3]. For many applications, which could give real-world impact; such as miniaturization with the integration of electronics, photonics interconnects as 
sensitive analytical devices [4]. Metals alloying is considered a way for developing new materials that have the best technological use as starting substances. Nanoparticles Alloy showed different physical and structural features than their bulk alloys [5]. The increase of solid solubility of the alloy components with particle size decrease is one of the prominent effects. Bimetallic nanoparticles (BMNPs) often improve the selectivity of metal-catalyzed reactions and excel monometallic nanocrystals; owing to their improved optical, electronic, and catalytic performances [6]. Furthermore, the change in metal composition retrofits another dimension in tailoring the BMNPs features alongside the shape and usual size manipulation [7]. Because of their extremely very small size with respectively large surface-to-volume ratio, the nano-sized materials serve as an ideal candidate for different applications. These features may be modified by varying size and shape, as well as composition through synthetic ways $[8,9]$. Raman signals' enhancement in SERS relays upon the electromagnetic field intensification for both incidents and scattered radiation in metal plasmon surfaces proximity as an electromagnetic enhancement, and/or on the non-resonant or resonant energy transfer between the adsorbed molecules and the metal nanoparticle as chemical enhancement [10]. Classically, since silver metal was demonstrated for inducing strong enhancement effects, these metal-based nanomaterial's have been used in SERS applications [11]; however, it was reported that silver nanostructures demonstrate a strong tendency to grow and/or reshape upon prolonged irradiation [12], in addition to its easy oxidation at room temperature [13]. For these reasons, the attention was focused on hybrid nanomaterial in recent years, where the features of two elements or more have been combined in a single platform [12]. Hybrid materials are more effective than the separated components and show emerging properties concerning the features derived from the summation of the starting elements [14]. The SERS effect was improved by combining different metals as core-shell structures and alloys [15] and as blending metals with polymers or metal oxides [16], to get multi-use nano-platforms in which plasmonic effects are enhanced [17]. Au and Ag could be combined in structures of hybrid bimetallic and core-shell formation; composed from two metals have already proved to be a good strategy for improving SERS efficiency [18]. In this work, an extensive study was carried out on the characteristics of nanophotonics SERS devices with mono and bimetallic alloying Au-Ag nanoparticles. The Si nano-columns were utilized as SERS substrates to detection of chlorpyrifos. The process of ion reduction of $\mathrm{Au}$ and $\mathrm{Ag}$ ions was employed to create the mono and bimetallic nanoparticles.

\section{THE EXPERIMENTAL WORK}

\section{Chemicals}

Hydrofluoric acid 40\%, (HIMEDIA), 99\% purity Chloroauric acid $\left(\mathrm{HAuCl}_{4} .3 \mathrm{H}_{2} \mathrm{O}\right), 99.99 \%$ silver nitrate $\left(\mathrm{AgNO}_{3}\right)$, and high purity $99.99 \%$ ethanol were purchased from Sigma Aldrich. $40 \mathrm{M}$ $\mathrm{HF}$ was diluted with ethanol to obtain the etching HF solution at $28 \mathrm{M}$ concentration. Chlorpyrifos, 0 , 0-diethyl 0-(3, 5, 6-trichloro-2-pyridinyl)-phosphorothioate was used to prepare the Chlorpyrifos solutions at concentrations of $25 \times 10^{-8}, 12 \times 10^{-7}, 6 \times 10^{-6}$, and $3 \times 10^{-5} \mathrm{M}$. All solutions were prepared by utilizing double-distilled water, and the chemicals were used, as received, without further purification.

\section{Formation Procedure of Si nano-column SERS-based Substrate.}

Si nano-columns samples; with preferred topographical features, were synthesized via pulsed laser-induced etching with $20 \%$ laser pulse duty cycles; using $500 \mathrm{~mW} / \mathrm{cm}^{2}$ intensity $(405 \mathrm{~nm})$ wavelength laser. The based bulk n-type Si substrate was oriented at (100) and having a resistivity of 3-5 $\Omega . \mathrm{cm}$. The synthesized Si nano-columns layer has high-density ion reduction centers (dangling bonds $\mathrm{Si}-\mathrm{H}$ ). This makes them very suitable to obtain high density and small dimensions hot spot junctions. The bimetallic substrates were cleaned by $10 \%$ HF diluted solution to eliminate any native oxide on their surfaces. The silicon substrates were fixed with two Teflon pieces and then immersed in the etching solution. Vertical pulsed diode laser etching of Si was obtained at $20 \%$ duty cycle and $10 \mathrm{~Hz}$ repetition frequency; see Figure 1. 


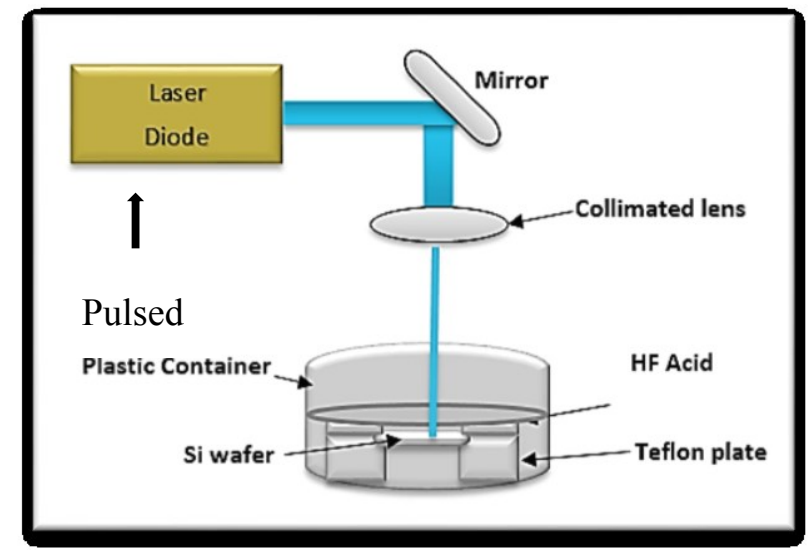

Figure 1: Experimental set-up of pulsed laser-induced etching process.

\section{Formation of nano photonics' SERS sensor}

The morphological control aspects of silicon nano-columns stratum can be utilized to optimize the performance of gold and silver nanoparticles/Si nano-columns SERS devices. The plasmonic characteristics of the active element $\mathrm{Au}$ and $\mathrm{Ag}$ nanoparticles change with dimensions of nanocolumns, shape, density, in addition to the surface roughness of the based substrate. The photonics SERS devices were fabricated through the activation of $\mathrm{Au}$ and $\mathrm{Ag}$ ions on silicon nano-columns through $\mathrm{Au}^{+3}$ and $\mathrm{Ag}^{+1}$ reduction to gold and silver nanoparticles by a rapid and simple immersion process. $\mathrm{Ag}-\mathrm{Au}$ NPs bimetallic alloys were obtained by immersing silicon nano-column layers in $\left(\mathrm{HAuCl}_{4}\right)$ and $\left(\mathrm{AgNO}_{3}\right)$ with $5 \times 10^{-3} \mathrm{M}$ solution concentrations; diluted few drops of $2.8 \mathrm{M} \mathrm{HF}$ within a dipping period of 2 and 5 min respectively at a room temperature. Au and Ag ions were reduced by the $\mathrm{Si}-\mathrm{Hx}(\mathrm{x}=1,2,3)$ groups of the columns stratum. The reaction pathway is illustrated hereunder $[19,20]$ :

$$
\begin{gathered}
\mathrm{Si}+6 \mathrm{HF} \rightarrow \mathrm{H}_{2} \mathrm{SiF}_{6}+4 \mathrm{H}^{+}+4 \mathrm{e} \\
\mathrm{Au}^{+3}+3 \mathrm{e}^{-} \rightarrow \mathrm{Au} \\
\mathrm{Ag}^{+1}+\mathrm{e}^{-} \rightarrow \mathrm{Ag}
\end{gathered}
$$

The concentration of the solution; required by individual nanoparticles, were obtained as [19]:

$$
\text { molarity }=\frac{\frac{W}{M \cdot W t}}{V}
$$

Where; W represents the $\mathrm{HAuCl}_{4}$ and $\mathrm{AgNO}_{3}$ weight in (g), M.Wt refers to $\mathrm{HAuCl}_{4}$ and $\mathrm{AgNO}_{3}$ molarity weights which are $\sim 339.8$ and $\sim 169.8731$ (g/mole) respectively. V refers to the volume of dissolved solution. Ion reduction of metallic ions and hence; the deposition of $\mathrm{Au} \mathrm{Ag}$ nanoparticles, occurs according to the reactions illustrated in figure 2. The fabricated bimetallic alloys nanophotonics SERS sensors, which have excellent hot spots junctions within the nanophotonics layer, were dipped in Chlorpyrifos pesticides solutions at $25 \times 10^{-8}, 12 \times 10^{-7}, 6 \times 10^{-6}$, and $3 \times 10^{-5} \mathrm{M}$ concentrations for $5 \mathrm{~min}$ and dried in air at room temperature before carrying out the SERS measurements. 


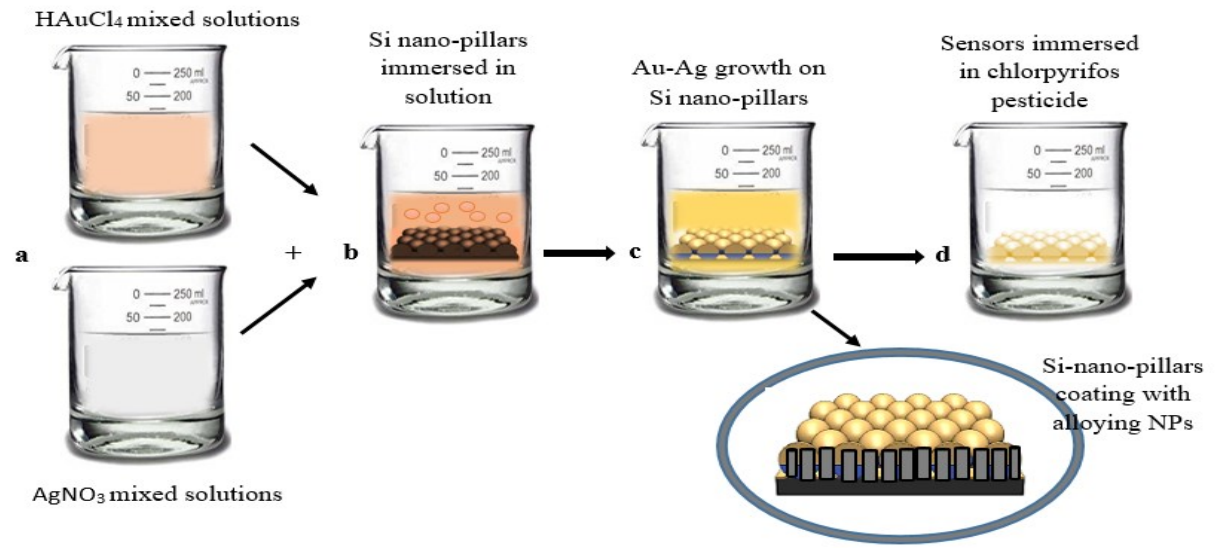

Figure 2: The formation of bimetallic alloys nanoparticles through ion reduction process.

\section{Instrumentation and data analysis}

The $\mathrm{Si}$ nano-columns surface, $\mathrm{Si}$ nano-columns/Au, $\mathrm{Si}$ nano-columns/Ag, and $\mathrm{Si}$ nanocolumns/Au-Ag nanoparticles nanophotonics pesticides sensors were characterized by (SPM) scanning probe microscope in contact mode; using 400 AFM system. The Morphological features of the nanophotonics sensors were utilized by Field-Emission scanning electron microscope FE-SEM (MIRA3 TESCAN) and X-ray diffraction (XRD-6000, Shemadzue). The bimetallic alloy nanoparticles sizes and the hotspot junction's dimensions were calculated by making use of the image J2 program. Raman and SERS spectra were recorded by an APUS TESCAN Raman microscope and the use of $10 \mathrm{~mW} ; 532 \mathrm{~nm}$ excitation DPSSND: YAG (CW) laser. The Raman peak intensity enhancement factor (EF) was worked out by considering the Chlorpyrifos molecule Raman peak, as shown in Equation 5, [21].

$$
\mathrm{EF}=\frac{I_{S E R S} \times C_{R}}{I_{R} \times C_{S E R S}}
$$

Where; IR and ISERS refer to the counterpart intensities of normal Raman and SERS. CR and CSERS and are the normal Raman and SERS concentrations of the probe respectively. The limit of detection (LOD) was determined following the dependency on the feature of the SERS peak and was utilized in Equation 6, [22].

$$
L O D=\frac{3 s_{d}}{d}
$$

Where; $S_{d}$ is the SERS intensity standard deviation for blank samples at Raman shift, and $d$ refers to the calibrated curve slope.

\section{RESULTS AND DISCUSSION}

\section{Silicon nano-columns, mono, and bimetallic nanoparticles characterization}

The altitudes, shapes, and surface roughness of the silicon nano-column layers were investigated by analyzing the 3-D micro AFM images. Figure (3a, b, and c), shows the microimages and histograms of silicon nano-columns, AuNPs, AgNPs, and Au-Ag NPs respectively. It illustrates uniform and high-density Si nano-columns over the Si surface. The profile depth of the pillars indicates a high degree of uniformity; with an average altitude of $54 \mathrm{~nm}$. The AuNPs, AgNPs and $\mathrm{Au}-\mathrm{Ag}$ NPs profiles show a large accumulation of particles that increase the surface roughness and reduce the depth of surface about $29,20 \mathrm{~nm}$, and $18 \mathrm{~nm}$ for gold, silver, and gold-silver respectively. The surface roughness was increased to $(4 \mathrm{~nm}),(6.7)$, and $(8.8 \mathrm{~nm})$ for gold, silver, and Au-Ag bimetallic nanoparticles correspondingly. These surface topographical properties variations are due to high-density nucleation sites and high-density metallic ions; resulted from the reduction process. The Si nano-columns sizes histogram, gold NPs, silver NPs, and gold-silver NPs are shown in figure 3. The sizes of aggregated monometallic nanoparticles in Ag are greater than those of gold and AuAg alloy nanoparticles. This is because the required electrons for reducing Ag are less than those needed by gold and Au-Ag alloy. 

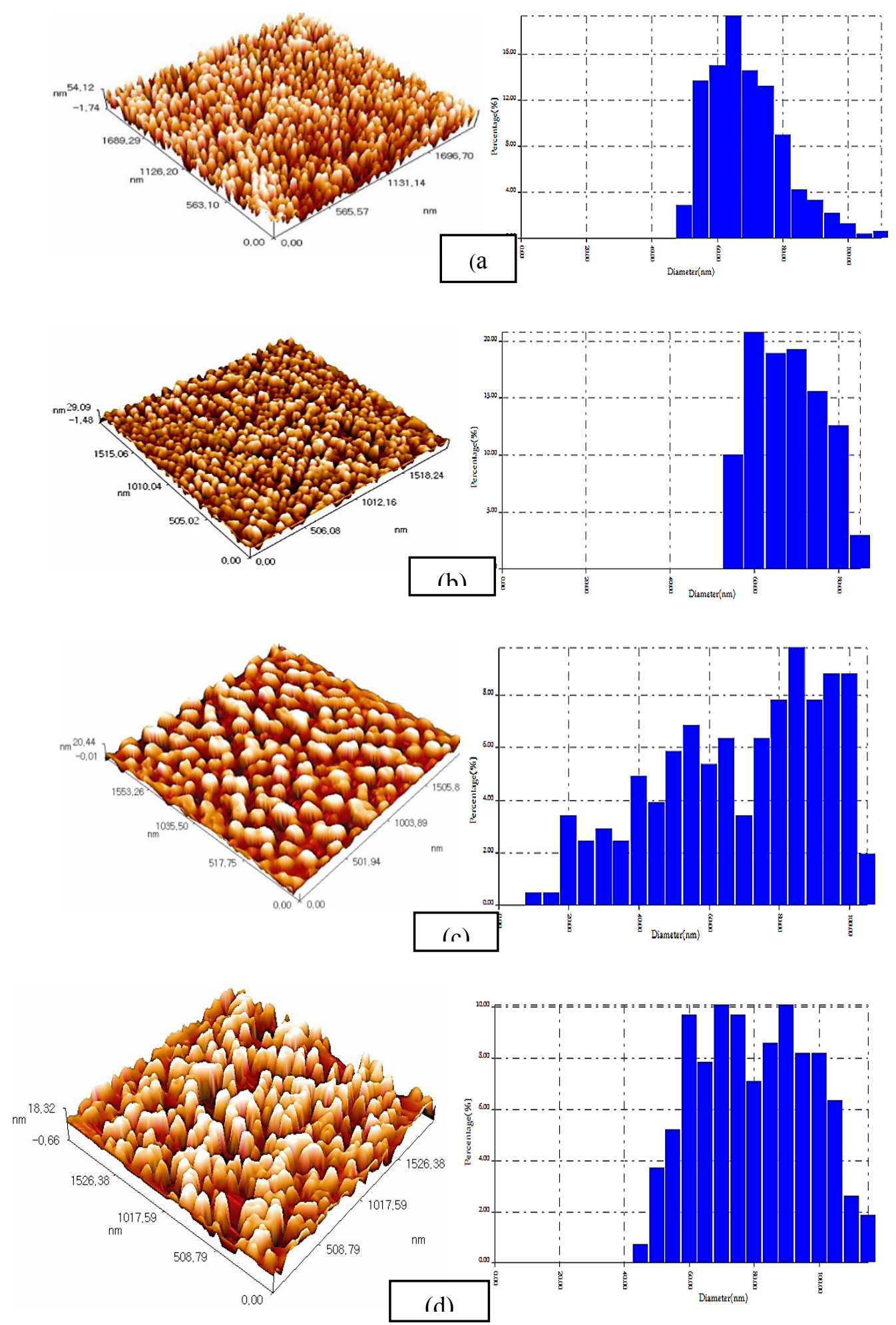

Figure 3: 3-D and histogram of silicon nano-columns size for (a) silicon nano-columns, (b) Au NPs and (c) Ag NPs (d) Au-Ag NPs

\section{Morphological aspects of nanophotonics SERS sensor.}

Bimetallic nanoparticles; deposited over silicon nano-columns were formed through the $\mathrm{Au}$ and $\mathrm{Ag}$ ions reduction with porous matrix dangling bonds. The $\mathrm{Au} \mathrm{NPs} / \mathrm{Si}$ nano-columns, $\mathrm{Ag} \mathrm{NPs} / \mathrm{Si}$ nano-columns, and Au-Ag NPs/Si nano-columns bimetallic alloy are illustrated in figure 4a, b, and $4 \mathrm{c}$ respectively. The size, density, and arrangement of the gold NPs, silver NPs, and gold-silver NPs depend on the morphology of the essential silicon nano-columns-based layer [8]. Figure 4 of the Field Emission Scanning Electron Microscope (FESEM) images show the nanoparticles gathered in a way that reflects the manner they were formed. 
There is a high aggregation tendency between mono and bimetallic nanoparticles; resulting in only one bimetallic layer. The evolution of the growth of the nanostructures can be observed for two nanomaterial types. Gold, silver, and gold-silver nano-aggregates were generated with a size range between 5-65, 35-315 nm, and from 4-40 nm as shown in Figure 5 (a, b, and c) respectively. The resulted hot spot junctions of the Au NPs, Figure 6a, in the range of 5-55 nm; within the highest peak at $25 \mathrm{~nm}$, permitted the generation of high-density nano-gaps in the plasmonic. For Ag nanoparticles; Figure $6 \mathrm{~b}$, the hot spot junctions are in the range of $25-150 \mathrm{~nm}$ with more high peaks at $100 \mathrm{~nm}$. This region represents hot and cold nano-gaps combinations while the hot spot junctions in Au-Ag NPs are in the range of 6-36 nm with more high peaks at $24 \mathrm{~nm}$. These categories represent hot nano-gaps combinations. Our findings conclude that the sizes of $\mathrm{Au}-\mathrm{Ag}$ alloy nanoparticles could be regarded as key-element of the silicon nano-columns features (altitude and surface roughness); where the greatest nucleation sites could contain the region of sharp nano-columns at high energy [23].
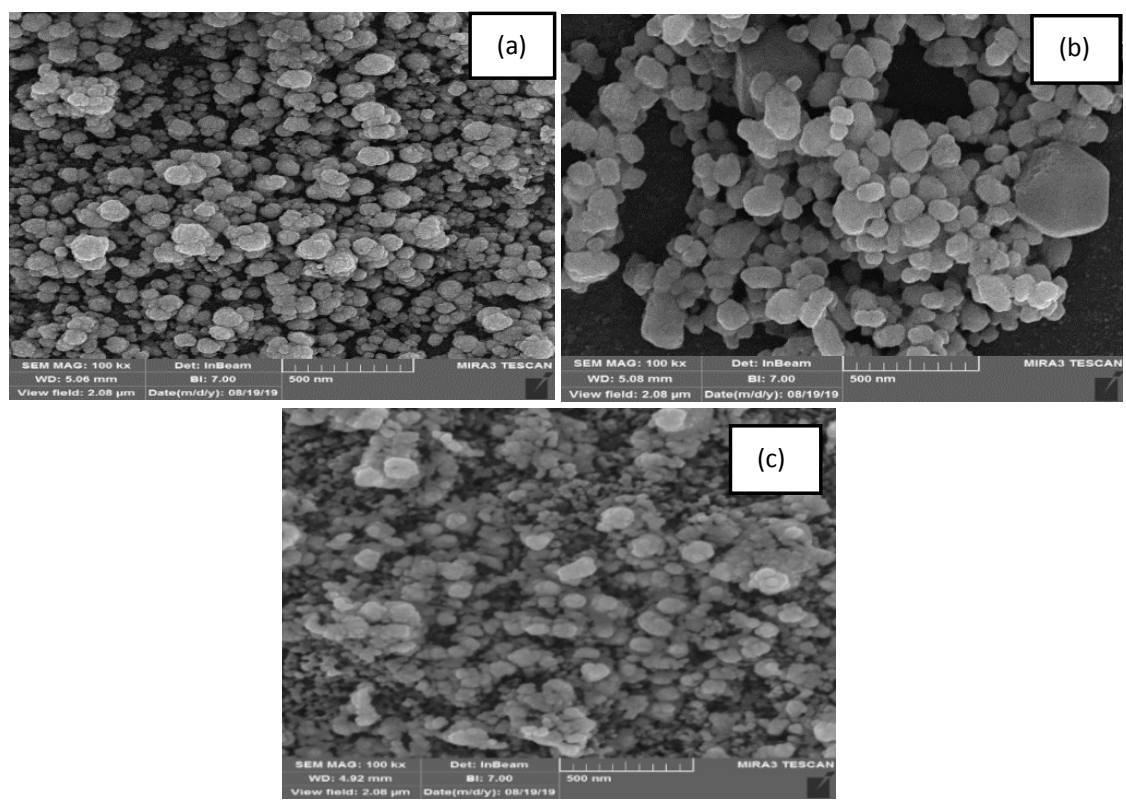

Figure 4: FE-SEM microimages of (a) Au NPs and (b) Ag NPs and (c) Au-Ag NPs.
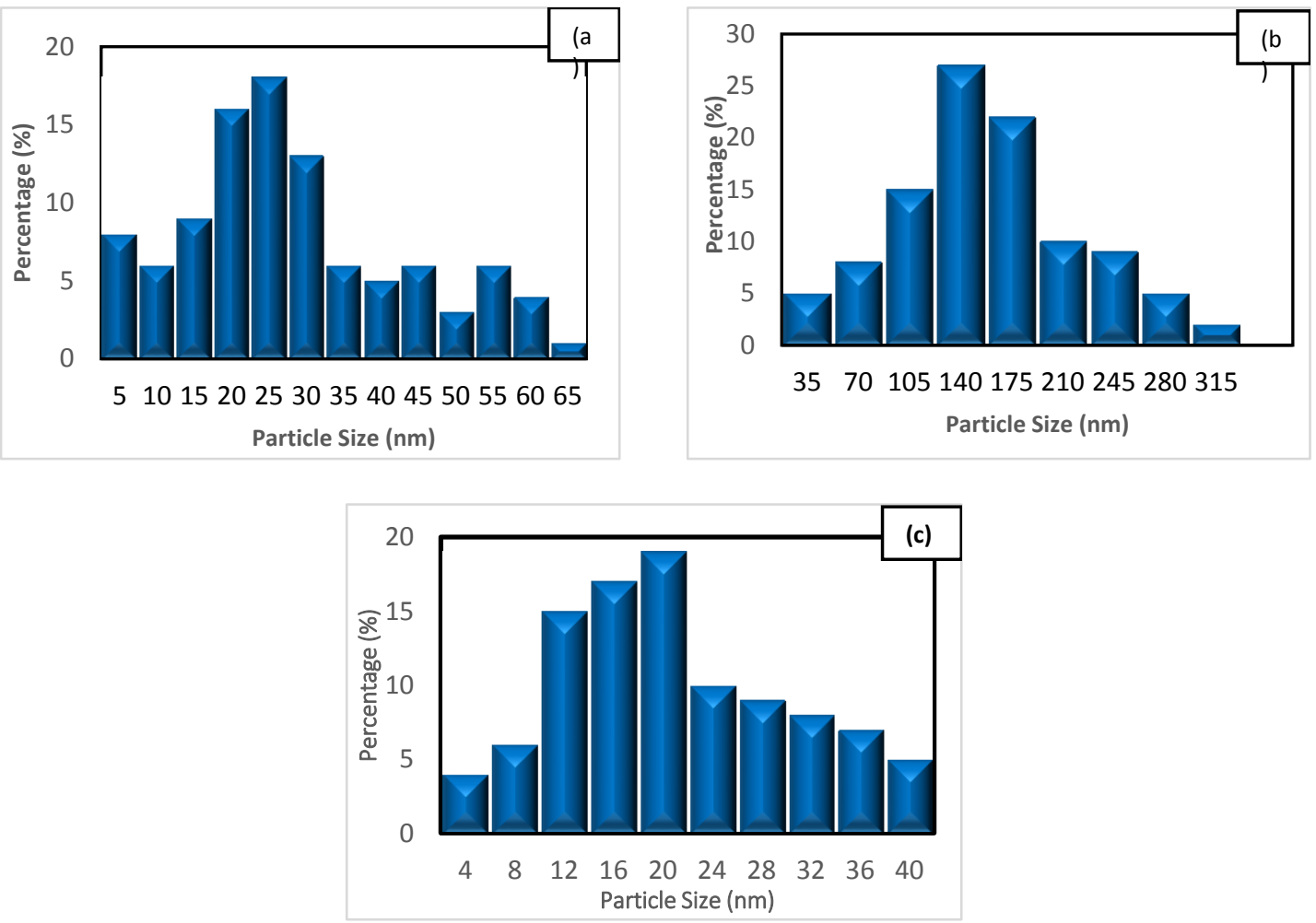

Figure 5: Histogram of particle size for (a) Au NPs and (b) Ag NPs and (c) Au-Ag NPs 

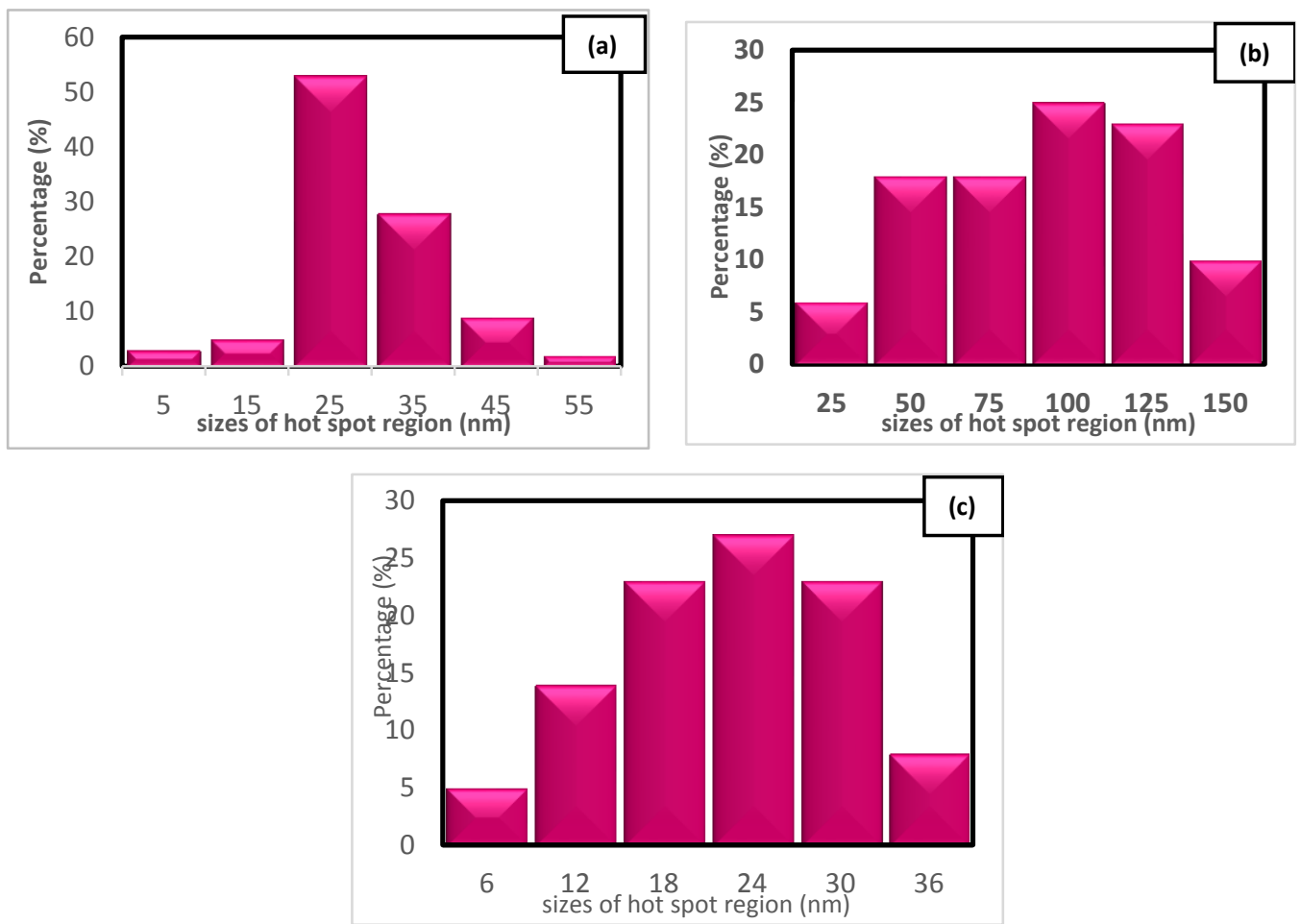

Figure 6: Histogram of hot spot region for (a) Au NPs and (b) Ag NPs and (c) Au-Ag NPs.

The EDX spectra of the SERS sensor with the mono gold or silver added to bimetallic alloy gold-silver nanoparticles deposited on Si nanopillar are illustrated in Fig.7 a, b, and c. The analysis of EDS has proved the existence of gold, silver, and silicon on the SERS sensor. The existence of silicon is from the substrate. It was found that the Au nanoparticles intensities are higher than for the Ag nanoparticles. This is due to the higher deposition rates of the Au NPs than the Ag NPs.

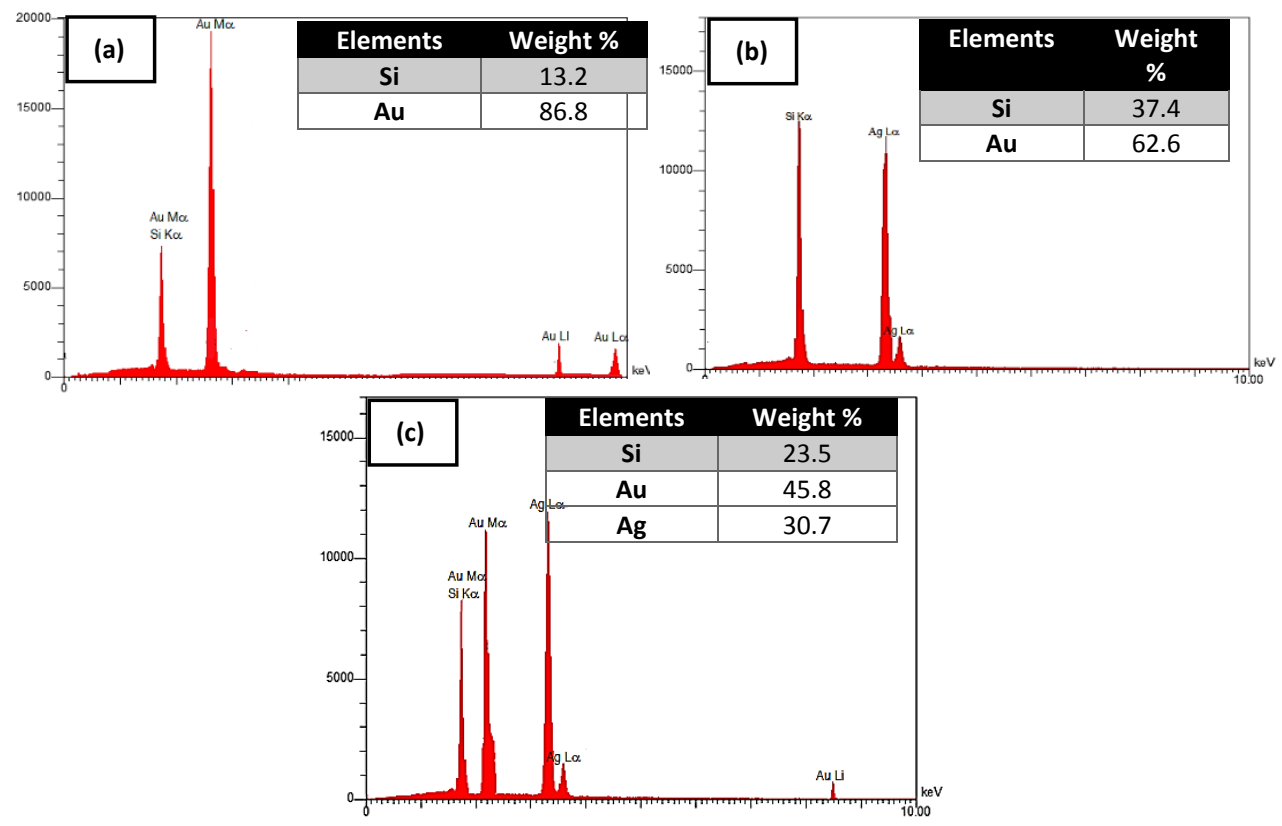

Figure 7: EDX of (a) Au NPs and (b) Ag NPs and (c) Au-Ag NPs

The XRD patterns show diffraction peaks of gold nanoparticles presented at $38.3^{\circ}$ and $44.2^{\circ}$ respectively and show diffraction peaks of silver nanoparticles located at $38.18^{\circ}$ and $44.3^{\circ}$ respectively as illustrated in Figure (8) a and b. Figure 8c shows diffraction peak patterns of gold nanoparticles presented at $39.02^{\circ}, 45.82^{\circ}$, and 64.52 respectively. These peaks are a combination of the individual peaks of Au and Ag elements and look very similar to the standard JCPDS file no.s 040783 and 01-1174, respectively. They suggest the formation of gold-silver bimetallic alloy 
nanoparticles [24]. The bimetallic nanoparticles' crystalline size was calculated by using the Shearers equation [25]. Bimetallic Au-Ag alloy nanoparticles have wider peaks than of monometallic Au and Ag nanoparticles; due to the very close values of lattice constants of gold (4.078) and silver (4.085) nanoparticles. This results in XRD peaks overlap of the gold-silver metallic nanoparticles [26]. The nanoparticles specific surface area (S.S.A) is given as [27]:

$$
\text { S.S. } A=\frac{6000}{\mathrm{D} * \rho}
$$

The monometallic Au and Ag density is (19.3 and 10.5) g/ $\mathrm{cm}^{3}$, correspondingly, and the $\mathrm{Au}-\mathrm{Ag}$ nanoparticles bimetallic alloys density (D), was determined by utilizing an equation that relies upon the EDS results in the analysis [28]:

$$
D=\frac{a * \rho+b * \rho}{a+b}
$$

Then, $\rho$ is the monometallic gold and silver nanoparticles density, D represents the density of Au-Ag bimetallic alloy nanoparticles in $\mathrm{g} / \mathrm{cm}^{3}$, an equal wt. \% Au and b is wt. \% Ag.
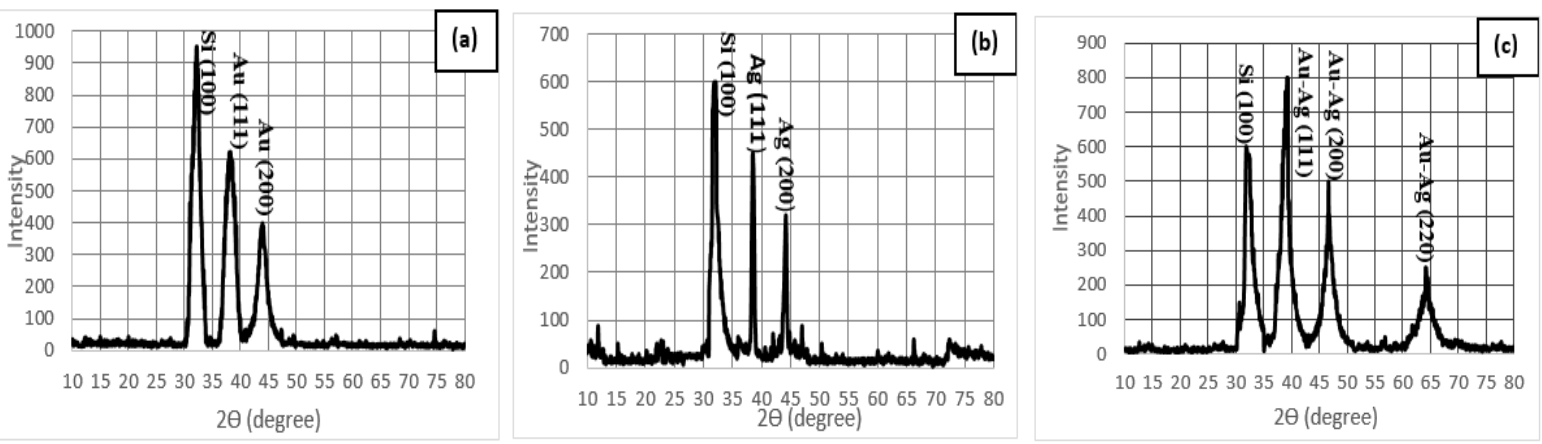

Figure 8: XRD of (a) Au NPs, (b) Ag NPs and (c) Au-Ag NPs

TABLE I: gold, silver and gold-silver nanoparticles FWHM, grain sizes and S.S.A. for different phases

\begin{tabular}{ccccc}
\hline \hline Phas & Nanoparticles & FWHM & Nanoparticle size & S.S.A \\
\hline \multirow{3}{*}{$(111)$} & $\mathrm{Au} \mathrm{NPs}$ & 0.066 & 2.22 & 140 \\
\cline { 2 - 5 } & $\mathrm{Ag} \mathrm{NPs}$ & 0.0110 & 13.37 & 42.7 \\
\cline { 2 - 5 } & $\mathrm{Au}-\mathrm{Ag} \mathrm{NPs}$ & 0.069 & 2.1 & 181 \\
\hline \multirow{3}{*}{$(200)$} & $\mathrm{Au} \mathrm{NPs}$ & 0.040 & 3.75 & 82.90 \\
\cline { 2 - 5 } & $\mathrm{Ag} \mathrm{NPs}$ & 0.0190 & 11.54 & 49.51 \\
\cline { 2 - 5 } & $\mathrm{Au}-\mathrm{Ag} \mathrm{NPs}$ & 0.051 & 2.9 & 131.2 \\
\hline (220) & $\mathrm{Au}-\mathrm{Ag} \mathrm{NPs}$ & 0.049 & 3.33 & 114 \\
\hline
\end{tabular}

\section{Sensing Performance of nanophotonics SERS pesticides sensors}

The Raman and SERS spectra features of the Chlorpyrifos pesticide sensors are illustrated in Figures 9a, b, and c. For the bare Si nano-columns layer without any metallic nanoparticles, the sensor performance for Chlorpyrifos solution showed a very weak Raman response; even at high (10$\left.{ }^{2} \mathrm{M}\right)$ Chlorpyrifos concentration. The intensity of the Raman peak increased dramatically after incorporating the $\mathrm{Au}-\mathrm{Ag}$ alloy nanoparticles on $\mathrm{Si}$ nano-columns. This has resulted from the plasmonics effects of bimetallic nanoparticles (hotspot junctions and nanoparticle sizes). The fabricated Si nano-columns with $\mathrm{Au}-\mathrm{Ag}$ alloy nanoparticles SERS pesticides sensors were tested under different Chlorpyrifos concentrations: $25 \times 10^{-8}, 12 \times 10^{-7}, 6 \times 10^{-6}$, and $3 \times 10^{-5} \mathrm{M}$. The enhanced Raman peaks in the SERS spectra of Chlorpyrifos is ascribed to C-C stretching, $\mathrm{P}-\mathrm{O}$ stretching, $\mathrm{P}=\mathrm{S}$ stretching, C-Cl stretching and P-O-R stretching bonds at wavenumbers $345 \mathrm{~cm}^{-1}, 536 \mathrm{~cm}^{-1}, 615 \mathrm{~cm}^{-}$ ${ }^{1}, 677 \mathrm{~cm}^{-1}$ and $1099 \mathrm{~cm}^{-1}$ [29] respectively. These Raman peaks are the fingerprint of Chlorpyrifos pesticide. In figure $9 \mathrm{~b}$, the lower performance of Ag nanoparticles is due to the lower population of hot spot junctions in the surface of Ag layer, in addition to the low specific surface area of the formed nanoparticles 


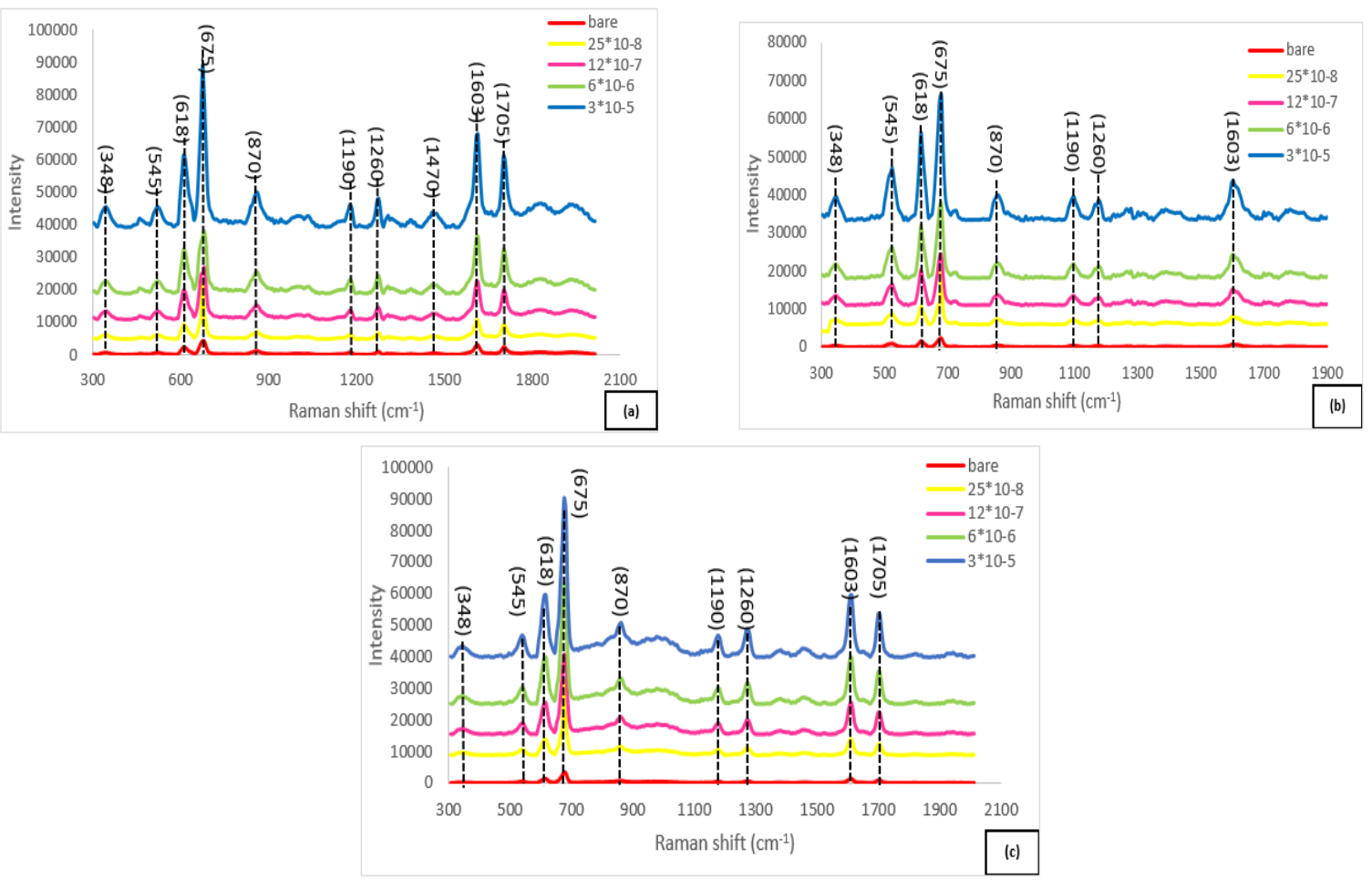

Figure 9: SERS spectra of chlorpyrifos with different concentrations from $25 \times 10^{-8}$ to $3 \times 10^{-5} \mathrm{M}$ for Si nano-columns/(a) Au (b) Ag and (c) Au-Ag nanophotonic sensors

To carry out a quantitative analysis of the fabricated nanophotonics pesticides sensors, a relationship between the characterized peak intensity $679 \mathrm{~cm}^{-1}$ of the SERS signal and the concentrations of Chlorpyrifos was considered and is illustrated in figure $10 \mathrm{a}, \mathrm{b}$, and c. This figure displays an exponential relationship within the concentration range $\left(3 \times 10^{-5}-25 \times 10^{-8} \mathrm{~mol} / \mathrm{L}\right)$ with a correlation coefficient (R2) of about 1, 0.96, and 0.99 for $\mathrm{Au}, \mathrm{Ag}$, and $\mathrm{Au}-\mathrm{Ag}$ nanoparticles on $\mathrm{Si}$ nano-columns respectively. The excellent exponential performance signifies the activity of the $\mathrm{Si}$ nano-columns nanophotonics SERS pesticides sensors to detect low pesticide concentrations. The principal reason for this attitude is related to the existence of hot spot regions existence of among the gold, silver, and gold-silver alloy nanoparticles and also to the specific surface area. An effective energy transfer may occur between the bimetallic nanoparticles and the pesticide molecules insides the hot spot junctions. This could enhance the detection efficiency of the sensors towards the pesticide and hence; strengthen the Raman signal. The most important parameter for assessing the activity of the sensors, in addition to its linearity, is the signal enhancement factor (EF) was calculated from equation (5). Figure 11 illustrates the EF variation with the concentration of Chlorpyrifos pesticides solution and shows the stable performance of the mono and bimetallic SERS sensors without any saturation effects. This means a continuous increase of the EF with the decrease of the pesticide concentration.

The highest value of EF of Chlorpyrifos $\left(1.54 \times 10^{6}\right)$ was obtained at ultralow $25 \times 10^{-8} \mathrm{~mol} / \mathrm{L}$ concentration with $\mathrm{Au}-\mathrm{Ag}$ alloy nanoparticles.
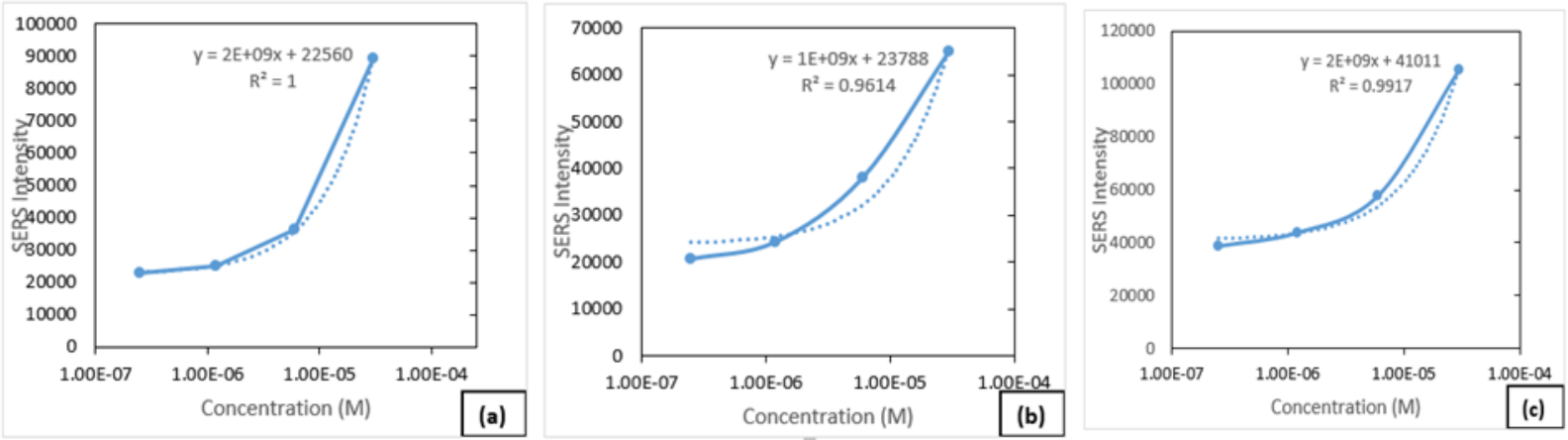

Figure 10: SERS intensity against Chlorpyrifos concentration at highest peak of $679 \mathrm{~cm}-1$ for $\mathrm{Si}$ nanophotonics SERS sensor for (a) Au (b) Ag and (c) Au-Ag 
The LOD of Chlorpyrifos (calculated from equation 3) for the sensors gold, silver, and goldsilver nanoparticles on Si nanocolumns and the obtained values for the gold, silver, and gold-silver nanoparticles is about $(0.07 \mathrm{ppm}),(0.3 \mathrm{ppm})$, and $(0.069 \mathrm{ppm})$ respectively. The obtained values in the present work are much lower than what'd been published recently [30]. This reflects the most significant point, that the application of bimetallic alloy nanoparticles provides an efficient way to detect extremely low concentrations of pesticides with higher enhancement factors.
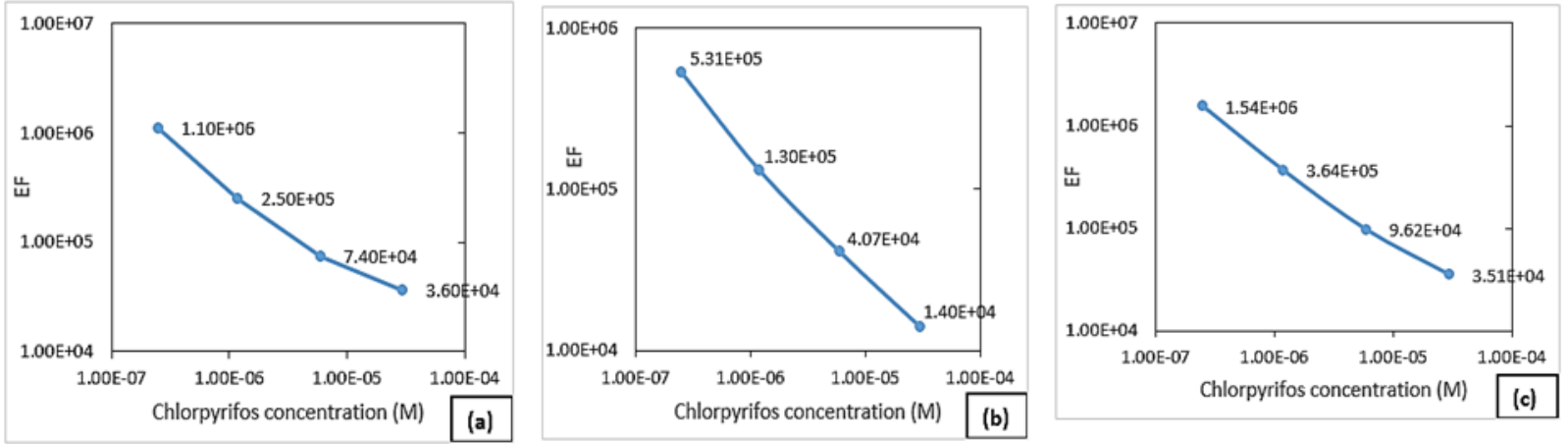

Figure 11: EF against Chlorpyrifos concentration at highest peak of $679 \mathrm{~cm}-1$ of nano photonics for (a)Au, (b) Ag and (c) Au-Ag

\section{Conclusion}

Nanophotonics SERS sensor was fabricated on the superior characteristics of based Si nanocolumns substrate and synthesized by the pulsed laser-induced photochemical method. The nanophotonics sensors with $\mathrm{Au}-\mathrm{Ag}$ alloy nanoparticles showed an extremely higher Raman enhancement factor $\left(1.56 \times 10^{6}\right)$ than that of monometallic $\mathrm{Au}$ and $\mathrm{Ag}$ nanoparticles. Very low concentrations of Chlorpyrifos pesticide have been successfully detected by utilizing nanophotonics SERS sensors with monometallic gold, silver, and bimetallic alloy gold-silver nanoparticles. The $\mathrm{Si}$ nano-columns layer provided a high density of small sizes nano-gaps hot spots junctions within the nanophotonics layer. The performance of these sensors was found to depend significantly on the density and size distribution of hotspot junctions, in addition to the high values of bimetallic nanoparticles specific surface. Efficient detection of trace values $(0.069 \mathrm{mg} / \mathrm{kg})$ LOD has been obtained when using the Au-Ag nanoparticles alloy in the fabrication of the nano photonics SERS sensors.

\section{References}

[1] H. Zhanghua and I. B. Sergey "Radiation guiding with surface plasmon polaritons" Rep. Prog. Phys., Vol. 76, pp.016402, 2013.

[2] V. A. Rivera, F. A. Ferri and E. Marega "Localized surface plasmon resonances: noble metal nanoparticle interaction with rare-earth ions" Plasmonics - Principles and Applications book, pp. 283-312, 2012.

[3] I. Malkiel, M. Mrejen and A. Nagler "Plasmonic nanostructure design and characterization via Deep Learning.” Light Sci Appl, Vol. 7, No. 60, 2018.

[4] L. W. Adam and C. B. Ryan "Photonics-on-a-chip: recent advances in integrated waveguides as enabling detection elements for real-world, lab-on-a-chip biosensing applications" Analyst, Vol. 136, No. 2, pp. 227-236, 2011.

[5] N.M. Ahmed, Y. Al-Douri, A.M. Alwan, A.A. Jabbar and G. E. Arif "Characteristics of nanostructure silicon photodiode using laser assisted etching" Procedia Engineering, Vol. 53, pp. 393-399, 2013.

[6] M. C. Manasi, N. G. Vinod and B. P. Vandana "Performance parameters and characterizations of nanocrystals: a brief review" Pharmaceutics, Vol. 8, No. 26, 2016.

[7] M. Kriener, M. Kamitani, T. Koretsune, R. Arita, Y. Taguchi, and Y. Tokura, "Tailoring band structure and band filling in a simple cubic (IV, III)-VI superconductor" Phys. Rev. Materials, Vol. 2, No. 4, pp. 044802, 2018.

[8] Ibrahim K., Khalid S. and Idrees K. "Nanoparticles: properties, applications and toxicities" Arabian Journal of Chemistry, Vol. 12, No. 7, pp. 908-931, 2019. 
[9] A. M. Alwan "Calculation of energy band gap of porous silicon based on the carrier transport mechanisms" Engineering and Technology Journal, Vol.25, pp. 1143-1148, 2007.

[10] D. Song-Yuan, Y. En-Ming, T. Zhong-Qun and M. Martin "Electromagnetic theories of surface-enhanced Raman spectroscopy” Chem. Soc. Rev., Vol. 46, pp. 4042-4076, 2017.

[11] D. A. Hashim, A. M. Alwan, and M.F. Jawad "Influence of Ag NPs on silicon nanocolumns NH3 gas sensors". Journal of the Electrochemical Society, Vol. 165, No. 14, pp.773-778, 2018.

[12] G. Marta, C. Elena, S. Paola and L. Loredana "Multilayer gold-silver bimetallic nanostructures to enhance SERS detection of drugs" Molecules, Vol. 25, pp. 3405, 2020.

[13] B. Fortuni, T. Inose, S. Uezono, S. Toyouchi, K. Umemoto, S. Sekine, Y. Fujita, M. Ricci, G. Lu, A. Masuhara and J.A. Hutchison "In situ synthesis of Au-shelled Ag nanoparticles on PDMS for flexible, long-life, and broad spectrum-sensitive SERS substrates” Chemical Communications, Vol. 53, pp. 11298-11301, 2017.

[14] M. Stefanos, M. P. Roger and T. K. Nguyen "Characterization techniques for nanoparticles: comparison and complementarity upon studying nanoparticle properties” Nanoscale, Vol. 10, pp. 12871-12934, 2018.

[15] T. H. Thi, H. V. Xuan, D. D. Nguyen, T. T. Tran, V. T. Nguyen, D. T. Tran, M. T. Pham and X. C. Nguyen "The structural transition of bimetallic $\mathrm{Ag}-\mathrm{Au}$ from core/shell to alloy and SERS application" RSC. Advances, Vol. 10, pp. 24577-24594, 2020.

[16] S. Sarkar, E. Guibal , F. Quignard and A. K. SenGupta "Polymer-supported metals and metal oxide nanoparticles: synthesis, characterization, and applications” Journal of Nanoparticle Research, Vol. 14, No. 715, 2012.

[17] U. Kosei and M. Hiroaki "Surface plasmon-enhanced photochemical reactions" Journal of Photochemistry and Photobiology C: Photochemistry Reviews, Vol. 15, pp. 31-52, 2013.

[18] A. M. Alwan, D. A. Hashim and M.F. Jawad "Optimizing of porous silicon alloying process with bimetallic nanoparticles” Gold Bulletin, Vol. 51, pp. 175-184, 2018.

[19] A. B. Dheyab, A. M. Alwan, and M. Q. Zayer, "Optimizing of Gold Nanoparticles on Porous Silicon Morphologies for a Sensitive Carbon Monoxide Gas Sensor Device” Plasmonics, Vol. 14, pp. 501-509, 2019.

[20] A.M. Alwan, A.J. Hayder, A.A. Jabbar "Study on morphological and structural properties of silver plating on laser etched silicon" Surface and Coatings Technology, Vol. 283, pp. 22-28, 2015.

[21] L. A. Wali, K. K. Hasan, M. A. Alwan, "An investigation of efficient detection of ultra-low concentration of penicillin in milk using AuNPs/PSi hybrid structure” Plasmonics, pp. 1-9, 2020.

[22] W. Kaiqiang, S. Da-Wen, P. Hongbin, and W. Qingyi "Surface-enhanced Raman scattering of core-shell Au@Ag nanoparticles aggregates for rapid detection of difenoconazole in grapes" Talanta, Vol. 191, pp. 449456, 2019.

[23] A. M. Alwan, M.F. Jawad and D. A. Hashim "Enhanced morphological properties of macroporous silicon with the incorporation of $\mathrm{Au}-\mathrm{Ag}$ bimetallic nanoparticles for improved $\mathrm{CO}_{2}$ gas sensing" Plasmonics, Vol. 14, pp. $1565-1575,2019$.

[24] E. Rodrigo, S. Alan, R. Alvaro, A. Alvaro, B. Daniel, M. Jose, L. Janet and P. Ramiro "Study of PtPd bimetallic nanoparticles for fuel cell applications" Materials Research, Vol. 20, No. 5, pp. 1193-1200, 2017.

[25] M. L. Francisco, M. M. Felipe, H. Paulo, F. Ana, and N. Francisco "Nanostructured titanium dioxide average size from alternative analysis of Scherrer's Equation” Matéria, Vol. 23, No. 1, 2018.

[26] W. Ke-Jun, G. Yunhu and T. Laura "Continuous synthesis of hollow silver-palladium nanoparticles for catalytic applications” Faraday Discuss., Vol. 208, pp. 427-441, 2018.

[27] A. H. Duaa, A. M. Alwan, and F. J. Muslim, "An investigation of structural properties of monometallic $(\mathrm{Ag}, \mathrm{Pd})$ and bimetallic (Ag@ Pd) nanoparticles growth on macro porous silicon" International Journal of nanoelectronics and material, Vol. 11, pp. 461-472, 2018.

[28] A. L. Wali, M. M. Alwan, A. B. Dheyab and D. A. Hashim“Excellent fabrication of Pd-Ag NPs/PSi photocatalyst based on bimetallic nanoparticles for improving methylene blue photocatalytic degradation" Optik - International Journal for Light and Electron Optics, Vol. 179, pp.708-717, 2019.

[29] W. K. Hamoudi, A. M. Alwan and D. A. Hashim "Controllable formation of plasmonic gold nanoparticles by pulsed laser-induced etching” Opt. Quant. Electron. Vol. 52, No. 351, 2020.

[30] Z. Chen, L. Yongyu, P. Yankun, and X. Tianfeng "Detection of chlorpyrifos in apples using gold nanoparticles based on surface enhanced Raman spectroscopy" International Journal of Agricultural and Biological Engineering, Vol. 8, No. 5, pp. 113-120, 2015. 